Original Research Paper

\title{
A Flexible, Real-Time Algorithm for Simulating Correlated Random Fields and Its Properties
}

\author{
Michael A. Kouritzin, Fraser Newton and Biao Wu \\ Department of Mathematical and Statistical Sciences, \\ University of Alberta, Edmonton (Alberta), Canada T6G 2 G1
}

Article history

Received: 12-6-2017

Revised: 28-07-2017

Accepted: 25-09-2017

Corresponding Author:

Michael A. Kouritzin

Department of Mathematical and Statistical Sciences,

University of Alberta,

Edmonton (Alberta), Canada

T6G 2G1

Email: michaelk@ualberta.ca

\begin{abstract}
Contemporary real-time problems like CAPTCHA generation and optical character recognition can be solved effectively using correlated random fields. These random fields should be produced on a graph in order that problems of any dimension and shape can be handled. However, traditional solutions are often too slow, inaccurate or both. Herein, the Quick Simulation Random Field algorithm to produce correlated random fields on general undirected graphs is introduced. It differs from prior algorithms by completing the graph and setting the unspecified covariances to zero, which facilitates analytic study. The Quick Simulation Random Field graph distribution is derived within and the following questions are studied: (1) For which marginal pmfs and covariances will this algorithm work? (2) When does the marginal property hold, where the sub-graph distribution of an algorithm-simulated field matches the distribution of the algorithm-simulated field on the subgraph? (3) When does the permutation property hold, where the vertex simulation order does not affect the joint distribution?
\end{abstract}

Keywords: Simulation, Correlated Random Field, Markov Random Field, Graph, Coupling, Permutation Property

\section{Introduction}

Correlated random fields are used in science and technology to model spatially distributed random objects. The applications of random field across the sciences are broad and include sequential Monte Carlo, computer vision, cryptography, astrophysics, rainfall, hydrology, analysis of gene expression time series, medical image processing and inverse optics and image synthesis; see, for example, Kouritzin (2017), Schlather et al. (2015), Chellappa and Jain (1993), Diaconis (2009), Vio et al. (2002), Leblois and Creutin (2013), Li et al. (2008), Li et al. (1995), Li (1995) and Winkler (2003). Furthermore, mathematicians often want to couple a collection of random variables with given distributions together on a single probability space while matching some constraint like covariances. In either situation, the complete joint distribution of the field may be unknown or even irrelevant as enough meaningful information is captured by marginal distributions and pairwise covariances between random variables. In the Gaussian case, many simple efficient methods, like covariance matrix decomposition, moving averages, Fast Fourier Transform (FFT), turning bands and local average subdivision, exist (see Shinozuka and Deodatis (1996),
Kleiber (2016) or Blanchard et al. (2016) for example). However, these methods are easiest to use over a regular grid and many random fields are fundamentally nonGaussian. In the general case, probability density functions are usually approximated by probability mass functions (pmfs) if necessary and some type of Markov chain Monte Carlo method is used when exact field distributions are desired. However, these methods require a very large number of iterations to converge, for example it took 2000 iterations in the simple Hamlet example in Diaconis (2009), and therefore are generally not suitable to real time computations. On the other hand, there are many approximation methods, often based upon the FFT or spectral decomposition and Karhunen-Loeve expansion to approximate covariance structure of fields (see e.g., Vio et al. (2002)).

To meet the diversity of problems in a variety of dimensions, Kouritzin et al. (2014) considered random fields on a general undirected graph structure and proposed an algorithm for producing a new class of discrete correlated random field on such graphs by either one-pass simulation or Gibbs-like resampling. The approach has been applied to Optical Character Recognition (OCR) Kouritzin et al. (2014) and the generation of both black-and-white Kouritzin et al. 
(2013) and gray-level Newton and Kouritzin (2011) CAPTCHAs (Fig. 1 shows a new example of such a gray level CAPTCHA.) The class of random fields created by their algorithm incorporate given probability mass functions (pmfs) at the vertices in a graph and specified pairwise covariances corresponding to edges existing in that graph. (This is translated into a pmf for the gray levels of each pixel and covariances between nearby pixels in this CAPTCHA example.) The joint distribution between pairs of vertices connected by a specified covariance edge is known in terms of two sets of auxiliary parameter pmf collections that can be selected for generality. However, the joint subgraph distribution on an incomplete subgraph is unknown for the algorithm in Kouritzin et al. (2013).

The starting point for the simulation consists of a fixed portion as well as a design portion. The fixed portion is an undirected graph together with the desired marginal vertex pmfs (the $\pi^{\prime} s$ ) and the collection of nonzero covariances (the $\beta^{\prime} s$ ) for the graph edges. (This setting is general enough to handle simulation in any dimension for example.) The design portion consists of two sets of auxiliary (vertex) pmfs (the $\hat{\pi}^{\prime} s$ and the $\tilde{\pi}^{\prime} s$ ) that can be used in place of the $\pi^{\prime} s$ in portions of the algorithm to do things like improve efficiency or destroy independence (Actually, there is a wide assortment of reasonable choices for the $\hat{\pi}^{\prime} s$ and the $\tilde{\pi}^{\prime} s$ discussed in Kouritzin et al. (2014)). Simulating the graph then amounts to directing the graph in an acyclic manner, fixing a topological sort of the vertices and using Proposition 1 of Kouritzin et al. (2014), requoted as Proposition 1 below, recursively (See Kouritzin et al. (2014) for details.) Our modified algorithm, introduced herein, completes the graph by adding edges of zero covariance wherever necessary before simulation. This completion does not complicate nor slow the simulation yet allows us to derive the complete field distribution in closed form for all possible auxiliary pmf parameters. We call this completed-graph simulation algorithm and resulting random field the quick simulation algorithm and quick simulation field herein.

This paper focuses on the constraints and properties of the random field generated by this quick simulation algorithm. Naturally, the algorithm cannot work for all possible parameters and might not work for others. We start by giving the joint (field) distribution of the random field generated by this algorithm (when it works). From there, we study regularity, meaning when the algorithms does provide a legitimate distribution over the whole space of vertices. This is equivalent to ensuring that the recursive formula (2.5) of Proposition 1 produces a conditional pmf in every iteration. It was observed in our CAPTCHA Kouritzin et al. (2013) and OCR Kouritzin et al. (2014) applications that the occasional illegitimate conditional pmf value outside $[0,1]$ can be replaced with a value inside without noticeable effect on the simulation.



Fig. 1. A gray-level CAPTCHA

However, it is still important to know when the only possible source of irregularity is numeric and not algorithmic. Next, we establish the marginality property that ensures the distribution of a random field on a subgraph projected from the random field constructed on the whole graph is the same as that for a random field constructed directly on this subgraph. Finally, we investigate the permutation property that makes sure the random field simulated from all topological sorts corresponding to the same complete undirected graph are the same in the sense of probability distribution. We establish necessary and sufficient conditions for this permutation property.

\section{Example 1}

Suppose we have the following complete undirected graph $G$ with vertices $v_{1}, v_{2}, v_{3}$,

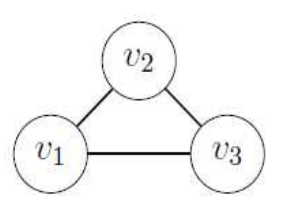

probability mass functions $\pi_{v_{i}}(-1)=\pi_{v_{i}}(1)=0.5, i=1,2,3$ and covariances $\beta_{v_{i} v_{j}}=0.1, i=1,2,3, j=1,2,3, i \neq j$. Let us illustrate the marginality and permutation properties of our algorithm.

Looking forward to (3.3), using the topological sort $v_{1}, v_{2}, v_{3}$ and setting $\hat{\pi}_{v_{i}}=\tilde{\pi}_{v_{i}}=\pi_{v_{i}}$ so $\tilde{g}\left(v_{i}, x_{v_{i}}\right)=\frac{x_{v_{i}}}{2}$ in (2.2) we assign the joint probabilities as follows:

\begin{tabular}{lccc}
\hline$x_{v_{1}}$ & $x_{v_{2}}$ & $x_{v_{3}}$ & $\Pi\left(X_{v_{1}}, X_{v_{2}}, X_{v_{3}}\right)$ \\
\hline 1 & 1 & 1 & 0.1625 \\
1 & 1 & -1 & 0.1125 \\
1 & -1 & 1 & 0.1125 \\
1 & -1 & -1 & 0.1125 \\
-1 & 1 & 1 & 0.1125 \\
-1 & 1 & -1 & 0.1125 \\
-1 & -1 & 1 & 0.1125 \\
-1 & -1 & -1 & 0.1625 \\
\hline
\end{tabular}


If we change the topologicial sort while maintaining all parameters, then we get the same joint pmf so the permutation property holds. It is straightforward to verify that the pmfs $\pi_{v_{i}}$ and covariances $\beta_{v_{i}, v_{j}}$ are as expected. Moreover, if we simulated two vertices $v_{i}, v_{j}$, then we get:

\begin{tabular}{lcl}
\hline$x_{v_{i}}$ & $x_{v_{j}}$ & $\Pi\left(X_{v_{i}}, X_{v_{j}}\right)$ \\
\hline 1 & 1 & 0.275 \\
1 & -1 & 0.225 \\
-1 & 1 & 0.225 \\
-1 & -1 & 0.275 \\
\hline
\end{tabular}

so marginality is also maintained.

In this note, we show how to compute these probabilities so that the pmfs and covariances are preserved in general as well as establish the conditions for the marginality and permutation properties above to hold.

The remainder of this note is laid out as follows: Section 2 contains our notation and background. Next, we give the closed form of correlated random field, discuss regularity and establish the marginality property in Section 3. The permutation property is studied in Section 4.

\section{Notation and Background}

\section{Probabilistic Setup}

Let $V$ be a finite set of vertices, $\vec{V}$ denote this set of vertices with an ordering and $\boldsymbol{X}_{v}$ be a finite state space for each $v \in V$. For any nonempty subsequence $\vec{B} \subset \vec{V}$, the space of configurations $x_{\vec{B}}=\left(x_{v}\right)_{v \in \vec{B}}$ on $\vec{B}$ is the Cartesian product $\mathcal{X}_{\vec{B}}=\prod_{v \in \vec{B}} \boldsymbol{X}_{v}$ and $\vec{B}^{C}$ denotes the subsequence so that $\vec{V}=\vec{B} \cup \vec{B}^{C}$. We abbreviate $\mathcal{X}_{\vec{V}}$ by $\mathcal{X}$ and $x_{v_{i}}$ by $x_{i}$ to ease notation. A random field $\Pi$ is a strictly positive probability measure on $\mathcal{X}$. The random vector $X=\left(X_{v}\right)_{v \in \vec{V}}$ on the probability space $\left(\mathcal{X}, 2^{\mathcal{X}}, \Pi\right)$ is also called a random field. For $\vec{B} \subset \vec{V}$, the random subfield on $\vec{B}$ is the projection map $X_{\vec{B}}: x \rightarrow x_{\vec{B}}$ from $\mathcal{X}$ onto $\mathcal{X}_{\vec{B}}$.

A neighborhood system $\partial=\{\partial(v): v \in V\}$ is a collection of subsets of $V$ :

- $v \notin \partial(v)$ for every $v \in V$

- $\quad v \in \partial(u)$ if and only if $u \in \partial(v)$

A random field $\Pi$ is Markov with respect to $\partial$ if for all $x \in \mathcal{X}$ :

$\Pi\left(X_{v}=x_{v} \mid X_{u}=x_{u}, u \neq v\right)=\prod\left(X_{v}=x_{v} \mid X_{\partial(v)}=x_{\partial(v)}\right)$

\section{Problem Statement}

Let $E$ be a set of edges where each $(u, v) \in E$ with $u$, $v \in V$ has no orientation but indicates $u, v$ are neighbors of each other. Then, $G=(V, E)$ is an undirected graph. If for every pair of vertices $u, v \in V$, there is a path of edges in $E$ connecting $u$ and $v$, then $G$ is connected. If every vertex in $G$ has a neighbor with at least two neighbors, then $G$ is sufficiently connected. If for every pair of nonneighbor vertices $z, u$ there is a neighbor of $z$ and a neighbor of $u$ that are distinct, then $G$ is disjoint pair rich. The open neighborhood of $\mathrm{v} \in V$ is $\partial_{G}(v)=\{u, u \neq v$, $(u, v) \in E\}$ and its closed neighborhood $\partial_{G}[v]=\partial_{G}(v) \cup\{v\}$. $\left\{\partial_{G}(v), v \in V\right\}$ is the neighborhood system implied by $G$. For any nonempty set $B \subset V$, the open neighborhood of $B$ is $\partial_{G}(B)=\bigcup_{v \in B} \partial_{G}(v) \backslash B$ and the closed neighborhood $\partial_{G}[B]=\partial_{G}(B) \cup B$. We set $\partial_{G}(\emptyset)=V$ for convenience.

We illustrate the new concepts of sufficiently connected and disjoint pair rich.

\section{Example 2}

Consider the graphs in Fig. 2.

Both graphs in Fig. 2 are connected. However, neither is sufficiently connected since in both cases none of the neighbors of $w$ have two neighbors.

\section{Example 3}

The graphs in Fig. 3 illustrate the definition of "disjoint pair rich".

In (B) non-neighbors $z$ and $u$ do not have distinct neighbors. Clearly in $(C)$, every vertex has a neighbor with two neighbors and every pair of non-neighbors has distinct neighbors. Yet, two vertices only have one neighbor.

\section{Example 4}

If every vertex in a graph $G$ has two neighbors, then it is disjoint pair rich. It is also sufficiently connected.

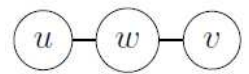

(a)

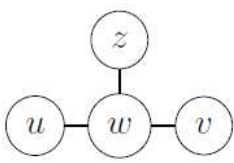

(b)
Fig. 2. Not sufficiently connected (a) Three vertex example (b) Four vertex example
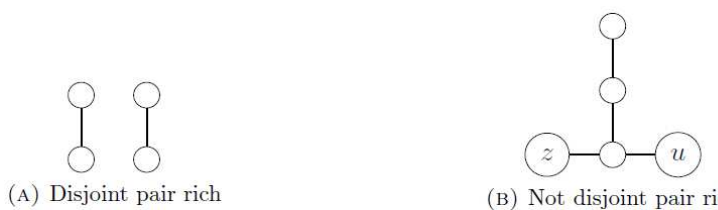

(B) Not disjoint pair rich

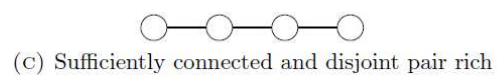

Fig. 3. Disjoint pair rich 
We are interested in creating a random field over $V$, where random variable $X_{v}$ at a vertex $v \in V$ has a predescribed pmf $\pi_{v}$ and random vectors $\left(X_{u}, X_{v}\right)$ have a predescribed non-zero covariance $\beta_{u v}\left(=\beta_{v u}\right)$ for each $(u$, $v) \in E$. Naturally, this problem could be ill-posed in the sense that there are mathematically incompatible collections of pmfs and covariances. Also, there often are multiple solutions with some being more efficient to simulate and others having nice properties like the marginal and permutation properties defined above.

\section{Directed Graph}

The random variables in the field are simulated in sequence. The first step towards sequencing is directing the graph. Let $A$ be a set of ordered vertex pairs, called arcs, (indicating the first vertex in the pair is simulated prior to the later). Then, $D=(V, A)$ is a directed graph. If $(u, v) \in A$ for $u, v \in V$, then, there is an arc from $u$ to $v ; u$ is a parent of $v$ and $v$ is a child of $u$. The set of parents of $v$ is denoted $\operatorname{pa}(v) . u$ is an ancestor of $v$ if there is a sequence of arcs from $u$ to $v . D$ is acyclic if there is no $v \in V$ that is an ancestor of itself.

\section{Graph Completion}

If $G=(V, E)$ is an undirected graph, then $\bar{G}=(V, \bar{E})$ denotes its completion, where there is an edge between every pair of vertices. Similarly, if $D=(V, A)$ is a directed graph, then $\bar{D}=(V, \bar{A})$ denotes its completion, where there is an arc between every pair of vertices and the direction of an arc that is also in $A$ matches that of A. Kouritzin et al. (2014) gives one possible algorithm to construct an acyclic complete directed graph $\bar{D}=(V, \bar{A})$ from a complete undirected graph $\bar{G}=(V, \bar{E})$ and a topological sort on $V$, i.e. a simulation order $\vec{V}=\left\{v_{i}\right\}_{i=1}^{N}$ where $N=|V|$ is the number of vertices. Our new Quick Simulation Algorithm works on a completed acyclic directed graph. Zero covariances are placed along any added arc i.e. $\beta_{v, u}=\operatorname{cov}\left(X_{u}, X_{v}\right)=0$ when $(v, u)$ or $(u, v)$ is in $\bar{A} \backslash A$.

\section{Conditional Probability Update}

The Quick Simulation Random Fields match a collection of pmfs $\left\{\pi_{v}, v \in V\right\}$ and a collection of covariances $\left\{\beta_{u v},(u, v) \in \bar{E}\right\}$. However, there are also two auxiliary pmf parameter sets $\left\{\hat{\pi}_{v}, \mathrm{v} \in V\right\}$ and $\left\{\tilde{\pi}_{v}, \mathrm{v} \in V\right\}$ that provide flexibility in the choice of field distribution as well as simulation. (See Kouritzin et al. (2014) for examples of choices for these auxiliary pmfs.) They also appear in the conditional probability update through functions:

$$
\tilde{g}\left(v, x_{v}\right)=\frac{\tilde{\pi}_{v}\left(x_{v}\right)\left(x_{v}-\tilde{\mu}_{v}\right\}}{\tilde{\sigma}_{v}^{2}}
$$

$$
\hat{h}(u, v)=\prod_{w \in p a(v) \backslash\{u\}} \hat{\pi}_{w}\left(x_{w}\right)
$$

for $\quad u \in \mathrm{pa}(v), \quad v \in V \quad$ and $\quad x_{v} \in \boldsymbol{X}_{v}, \quad$ where $\tilde{\mu}_{v}=\sum_{x_{v} \in X_{v}} \tilde{\pi}_{v}\left(x_{v}\right) x_{v}$ and $\tilde{\sigma}_{v}^{2}=\sum_{x_{v} \in X_{v}} \tilde{\pi}_{v}\left(x_{v}\right)\left(x_{v}-\tilde{\mu}_{v}\right)^{2} . \tilde{g}$ and $\hat{h}$ may look mysterious here. However, looking ahead to (3.3), we see they affect the field distribution in our new algorithm. $\tilde{g}$ normalizes the sample $x_{v}$ by subtracting the mean and dividing by the variance but it allows this normalization to be done with respect to any convenient non-trivial pmf $\tilde{\pi}$ that could be different than $\pi$. $\hat{h}$ allows us to consider all the parents except the one we are currently setting the covariance for as if they came from a different distribution $\hat{\pi}$. Intuitively, this makes sense. When we are focused on the covariance for one parent the other parents could have just as easily come from $\pi$ or $\hat{\pi}$. The following proposition establishes that this flexibility is allowed.

Let $\left\{v_{i}\right\}_{i=1}^{N}$ be a topological sort of directed graph $D$ $=(V, A)$. For any $B \subset V$, we let $j=\max \left\{i: 1 \leq i \leq N, v_{i} \in B\right\}$ and find:

$$
\begin{aligned}
& \Pi\left(X_{B}=x_{B}\right)= \\
& \sum_{x_{v_{k}}: \leq k \leq j, v_{k} \notin B} \prod_{i=1}^{j} \Pi\left(X_{v_{i}}=x_{v_{i}} \mid X_{p a\left(v_{i}\right)}=x_{p a\left(v_{i}\right)}\right)
\end{aligned}
$$

where, $\quad \Pi\left(X_{v_{1}}=x_{v_{1}} \mid X_{p a\left(v_{1}\right)}=x_{p a\left(v_{1}\right)}\right)=\Pi\left(X_{v_{1}}=x_{v_{1}}\right) . \quad$ The main proposition in Kouritzin et al. (2014) is:

\section{Proposition 1}

Assume that $D=(V, A)$ is a directed acyclic graph with $N$ vertices, $\left\{v_{i}\right\}_{i=1}^{N}$ is a topological sort of the vertices $V$ and $\left\{\tilde{\pi}_{v}\left(x_{v}\right): x_{v} \in \boldsymbol{X}_{v}, v \in V\right\},\left\{\hat{\pi}_{v}\left(x_{v}\right): x_{v} \in \boldsymbol{X}_{v}, v \in V\right\}$ are sets of auxiliary non-trivial pmfs. Suppose further that $\left\{\pi_{v}\left(x_{v}\right)\right.$ : $\left.x_{v} \in \boldsymbol{X}_{v}, v \in V\right\}$ are pmfs and $\left\{\beta_{v, u}:(u, v) \in A\right.$ or $\left.(v, u) \in A\right\}$ are numbers such that the right hand side of:

$$
\begin{aligned}
& \Pi\left(X_{v_{i}}=x_{v_{i}} \mid X_{p a\left(v_{i}\right)}=x_{p a\left(v_{i}\right)}\right) \\
& =\pi_{v_{i}}\left(x_{i}\right)+\frac{\tilde{g}\left(v_{i}, x_{i}\right)}{\prod\left(X_{p a\left(v_{i}\right)}=x_{p a\left(v_{i}\right)}\right)} \\
& \sum_{u \in p a\left(v_{i}\right)} \beta_{u, v_{i}} \tilde{g}\left(u, x_{u}\right) \hat{h}\left(u, v_{i}\right)
\end{aligned}
$$

is non-negative for each $x_{i} \in \boldsymbol{X}_{v_{i}}$ and $x_{p a\left(v_{i}\right)} \in \boldsymbol{X}_{p a\left(v_{i}\right)}$ $(1 \leq i \leq N)$, where $\Pi\left(X_{p a\left(v_{i}\right)}=x_{p a\left(v_{i}\right)}\right) \quad$ is computed according to (2.4). Form the conditional probabilities recursively using (2.5), starting with $\Pi\left(X_{v_{1}}=x_{1}\right)=\pi_{v_{1}}\left(x_{1}\right)$. Then, the random field $X$, defined by (the multiplication rule and (2.5)): 


$$
\Pi(X=x)=\prod_{i=1}^{N} \Pi\left(X_{v_{i}}=x_{i} \mid X_{p a\left(v_{i}\right)}=x_{p a\left(v_{i}\right)}\right)
$$

has marginal probabilities $\left\{\pi_{v}\right\}$ and covariances $\operatorname{cov}\left(X_{u}\right.$, $\left.X_{v}\right)=\beta_{v, u}$ for all $u \in p a(v)$.

\section{Remark 1}

The term non-trivial pmfs can be interpreted as: Each $\tilde{\pi}_{v}$ should have non-zero variance and each $\hat{\pi}_{v}$ should be strictly positive. These auxiliary pmfs affect the field distribution but not its marginal vertex pmfs nor its vertex-vertex covariances.

\section{Remark 2}

In Kouritzin et al. (2014), there was the stronger constraint that the right hand side of $(2.5)$ is in $[0,1]$. However, $\quad \sum_{x_{v} \in X_{v}} \frac{\tilde{g}\left(v, x_{v}\right)}{\Pi\left(X_{p a(v)}=x_{p a(v)}\right)} \sum_{u \in p a(v)} \beta_{u, v} \tilde{g}\left(u, x_{u}\right) \hat{h}(u, v)=0$ since $\sum_{x_{v} \in X_{v}} \tilde{g}\left(v, x_{v}\right)=\sum_{x_{v} \in X_{v}} \frac{\tilde{\pi}_{v}\left(x_{v}\right)\left(x_{v}-\tilde{\mu}_{v}\right)}{\tilde{\sigma}_{v}^{2}}=0$. Hence, if the right hand side of (2.5) is non-negative, then it is in [0, 1] and (2.5) defines a legitimate conditional pmf.

\section{Remark 3}

Notice that (2.5) gives the same value, whether we consider the given graph $D$ or its completion $\bar{D}$ where the added arcs have zero covariance.

\section{Distribution and Marginality of Quick Simulation Fields}

Proposition 1 can be extended to give the full field distribution when the graph is complete.

\section{Proposition 2}

Assume that $\bar{D}=(V=\bar{A})$ is a complete directed acyclic graph with $N$ vertices, $\vec{V}=\left\{v_{i}\right\}_{i=1}^{N}$ is a topological sort of the vertices $V$ and $\left\{\tilde{\pi}_{v}\left(x_{v}\right): x_{v} \in \boldsymbol{X}_{v}, v \in V\right\},\left\{\hat{\pi}_{v}\left(x_{v}\right): x_{v} \in \boldsymbol{X}_{v}, v \in V\right\} \quad$ are auxiliary non-trivial pmf sets. Suppose further that $\left\{\pi_{v}\left(x_{v}\right): x_{v} \in \boldsymbol{X}_{v}, v \in V\right\} \quad$ are $\quad$ pmfs and $\left\{\beta_{v, u}:(u, v) \in \bar{A}\right.$ or $\left.(v, u) \in \bar{A}\right\}$ are numbers such that the right hand side of:

$$
\begin{aligned}
& \prod\left(\mathrm{X}_{v_{i}}=x_{i} \mid \mathrm{X}_{v_{1}}=x_{1}, \ldots, \mathrm{X}_{v_{i-1}}=x_{i-1}\right)= \\
& \pi_{v_{i}}\left(x_{i}\right)+\frac{\tilde{g}\left(v_{i}, x_{i}\right) \sum_{j=1}^{i-1} \beta_{v_{j}, v_{i}} \tilde{g}\left(v_{j}, x_{j}\right) \hat{h}\left(v_{j}, v_{i}\right)}{\prod\left(\mathrm{X}_{v_{1}}=x_{1}, \ldots, \mathrm{X}_{v_{i-1}}=x_{i-1}\right)}
\end{aligned}
$$

is non-negative for each $x_{i} \in \boldsymbol{X}_{v_{i}}, i=1, \ldots, N$. Form the conditional probabilities recursively using (3.1), starting with $\Pi\left(X_{v_{1}}=x_{1}\right)=\pi_{v_{1}}\left(x_{1}\right)$. Then, the random field $X$, defined by:

$$
\prod(\mathrm{X}=x)=\prod_{i=1}^{N} \Pi\left(X_{v_{i}}=x_{i} \mid \mathrm{X}_{v_{1}}=x_{1}, \ldots, \mathrm{X}_{v_{i-1}}=x_{i-1}\right)
$$

- Has marginal probabilities $\left\{\pi_{v}\right\}$ and covariances $\operatorname{cov}\left(X_{u}, X_{v}\right)=\beta_{v, u}$ for all $u, v \in V$

- Has closed form

$$
\begin{gathered}
\prod_{v_{1}, \ldots, v_{n}}\left(x_{1}, \ldots, x_{n}\right)=\prod_{i=1}^{n} \pi_{v_{i}}\left(x_{i}\right) \\
+\sum_{1 \leq j<i \leq n}\left(\prod_{k=1, k \neq j}^{i-1} \hat{\pi}_{v_{k}}\left(x_{k}\right) \times \prod_{k=i+1}^{n} \pi_{v_{k}}\left(x_{k}\right)\right) \\
\tilde{g}\left(v_{i}, x_{i}\right) \beta_{v_{i}, v_{j}} \tilde{g}\left(v_{j}, x_{j}\right)
\end{gathered}
$$

for each $x_{i} \in \boldsymbol{X}_{v_{i}}$ and $n=1, \ldots, N$.

\section{Remark 4}

The one-pass algorithm (as opposed to the Gibbstype algorithm used in Kouritzin et al. (2013)) follows from (3.1). We just use the conditional probability to simulate the new vertex given the prior ones in the topological sort. However, the big efficiency comes from the fact that the terms in (3.1) are only non-zero (and hence need to be computed) in the case where $v_{j}$ is a parent of $v_{i}$ in the original (non-completed) graph.

\section{Remark 5}

Since the terms with $\beta_{v_{i}, v_{j}}=0$ disappear, the computations are the same as for the algorithm in Kouritzin et al. (2014) on the incomplete graph.

\section{Remark 6}

Regularity means that the right hand side of (3.1) is a conditional pmf. As noted in Remark 2, the right hand side of (3.1) need only be non-negative, which is equivalent to:

$$
\begin{gathered}
-\tilde{\sigma}_{v_{i}}^{2} \pi_{v_{i}}\left(x_{i}\right) \prod\left(\mathrm{X}_{v_{1}}=x_{1}, \ldots ., \mathrm{X}_{v_{i-1}}=x_{i-1}\right) \leq \\
\left(x_{i}-\tilde{\mu}_{v_{i}}\right) \sum_{j=1}^{i-1} \beta_{v_{j}, v_{i}} \tilde{g}\left(v_{j}, x_{j}\right) \hat{h}\left(v_{j}, v_{i}\right)
\end{gathered}
$$

and can be checked during the iteration. Notice:

(1) There is no constraint on $\beta_{v_{i}, v_{j}}$ when

$$
x_{i}=\tilde{\mu}_{v_{i}} \text { or } x_{j}=\tilde{\mu}_{v_{j}}
$$

(2) $\beta_{v_{i}, v_{j}}=0$ automatically satisfies the constraint

By (2), we need only check: 


$$
\begin{aligned}
& -\tilde{\sigma}_{v_{i}}^{2} \pi_{v_{i}}\left(x_{i}\right) \prod\left(\mathrm{X}_{v_{1}}=x_{1}, \ldots . ., \mathrm{X}_{v_{i-1}}=x_{i-1}\right) \\
& \leq\left(x_{i}-\tilde{\mu}_{v_{i}}\right) \quad \sum_{u \in p a\left(v_{i}\right)} \beta_{u, v_{i}} \tilde{g}\left(u, x_{u}\right) \hat{h}\left(u, v_{i}\right)
\end{aligned}
$$

where, $\operatorname{pa}\left(v_{i}\right)$ denotes parents in the original (notcompleted) graph. If $p a\left(v_{\mathrm{i}}\right)=\left\{v_{i-1}\right\}$ is a singleton, then (3.5) further simplifies by (2.2) to:

$$
-\tilde{\sigma}_{v_{i}}^{2} \tilde{\sigma}_{v_{i-1}}^{2} \frac{\pi_{v_{i}}\left(x_{i}\right) \pi_{v_{i-1}}\left(x_{i-1}\right)}{\tilde{\pi}_{v_{i}}\left(x_{i}\right) \tilde{\pi}_{v_{i-1}}\left(x_{i-1}\right)} \leq\left(x_{i}-\tilde{\mu}_{v_{i}}\right) \beta_{v_{i-1}, v_{i}}\left(x_{i-1}-\tilde{\mu}_{v_{i-1}}\right)
$$

for $x_{i} \in \boldsymbol{X}_{v_{i}}, x_{i-1} \in \boldsymbol{X}_{v_{i-1}}$. One can check (3.5) or (3.6) iteratively to ensure the Quick Simulation algorithm is producing a field with the desired pmfs and covariances. Now, we show how equality in (3.6) is hit:

$$
\begin{aligned}
& -\tilde{\sigma}_{v_{i}}^{2} \tilde{\sigma}_{v_{i-1}}^{2} \frac{\pi_{v_{i}}\left(x_{i}\right) \pi_{v_{i-1}}\left(x_{i-1}\right)}{\tilde{\pi}_{v_{i}}\left(x_{i}\right) \tilde{\pi}_{v_{i-1}}\left(x_{i-1}\right)}=\left(x_{i}-\tilde{\mu}_{v_{i}}\right) \beta_{v_{i-1}, v_{i}}\left(x_{i-1}-\tilde{\mu}_{v_{i-1}}\right) \\
& \Leftrightarrow \pi_{v_{i}}\left(x_{i}\right)=\pi_{v_{i}}\left(x_{i}\right)\left(1-\pi_{v_{i-1}}\left(x_{i-1}\right)\right) \\
& -\tilde{g}\left(v_{i-1}, x_{i-1}\right) \beta_{v_{i}, v_{i-1}} \tilde{g}\left(v_{i}, x_{i}\right) \\
& \Leftrightarrow \pi_{v_{i}}\left(x_{i}\right)=\sum_{y \neq x_{i-1}}\left(\begin{array}{l}
\pi_{v_{i}}\left(x_{i}\right) \pi_{v_{i-1}}(y)+\tilde{g}\left(v_{i-1}, y\right) \\
\beta_{v_{i}, v_{i-1}} \tilde{g}\left(v_{i}, x_{i}\right)
\end{array}\right) \\
& \Leftrightarrow \pi_{v_{i}}\left(x_{i}\right)=\prod\left(\mathrm{X}_{v_{i-1}} \neq x_{i-1}, \mathrm{X}_{v_{i}}=x_{i}\right)
\end{aligned}
$$

since $\sum_{y \neq x_{i}-1} \tilde{g}\left(v_{i-1}, y\right)=0$ and it is shown in Proposition 1 of Kouritzin et al. (2014) that $\Pi\left(X_{v_{i-1}}=x_{i-1}, X_{v_{i}}=x_{i}\right)$ $=\pi_{v_{i}}\left(x_{i}\right) \pi_{v_{i-1}}\left(x_{i-1}\right)+\tilde{g}\left(v_{i-1}, x_{i-1}\right) \beta_{v_{i}, v_{i-1}} \tilde{g}\left(v_{i}, x_{i}\right)$.

Hence, we hit this bound when we have a singleton parent and one value of $X_{i-1}$ precludes another value of $X_{i}$.

\section{Proof of Proposition 2}

a) This follows immediately from Proposition 1 and the fact that the parents of $v_{\mathrm{i}}$ are all $v_{1}, \ldots, v_{i-1}$ when the graph is complete.

b) Note (3.3) holds for $n=1$. Now, we assume it is true for $n-1$ with some $n \in\{2, \ldots, N\}$ and show it for $n$. (3.1) is equivalent to:

$$
\begin{gathered}
\Pi_{v_{1}, \ldots, v_{n}}\left(x_{1}, \ldots, x_{n}\right)=\pi_{v_{n}}\left(x_{n}\right) \prod_{v_{1}, \ldots, v_{n-1}}\left(x_{1}, \ldots, x_{n-1}\right) \\
\quad+\sum_{j=1}^{n-1}\left(\tilde{g}\left(v_{n}, x_{n}\right) \beta_{v_{j}, v_{n}} \tilde{g}\left(v_{j}, x_{j}\right) \prod_{k=1, k \neq j}^{n-1} \hat{\pi}_{v_{k}}\left(x_{k}\right)\right)
\end{gathered}
$$

so by (3.8) and (3.3) with $n-1$ :

$$
\begin{aligned}
& \Pi_{v_{1}, \ldots, v_{n}}\left(x_{1}, \ldots, x_{n}\right) \\
& =\pi_{v_{n}}\left(x_{n}\right) \prod_{i=1}^{n-1} \pi_{v_{i}}\left(x_{i}\right)+\sum_{j=1}^{n-1}\left(\begin{array}{c}
\tilde{g}\left(v_{n}, x_{n}\right) \beta_{v_{n}, v_{j}} \tilde{g}\left(v_{j}, x_{j}\right) \\
\prod_{k=1, k \neq j}^{n-1} \hat{\pi}_{v_{k}}\left(x_{k}\right)
\end{array}\right)
\end{aligned}
$$

$+\pi_{v_{n}}\left(x_{n}\right) \sum_{1 \leq j<i \leq n-1}\left[\begin{array}{l}\left.\left(\prod_{k=1, k \neq j}^{i-1} \hat{\pi}_{v_{k}}\left(x_{k}\right) \times \prod_{k=i+1}^{n-1} \pi_{v_{k}}\left(x_{k}\right)\right)\right] \\ \tilde{g}\left(v_{i}, x_{i}\right) \beta_{v_{i}, v_{j}} \tilde{g}\left(v_{j}, x_{j}\right)\end{array}\right]$

$=\prod_{i=1}^{n} \pi_{v_{i}}\left(x_{i}\right)$

$+\sum_{1 \leq j<i \leq n-1}\left[\begin{array}{l}\left(\prod_{k=1, k \neq j}^{i-1} \hat{\pi}_{v_{k}}\left(x_{k}\right) \times \prod_{k=i+1}^{n} \pi_{v_{k}}\left(x_{k}\right)\right) \\ \tilde{g}\left(v_{i}, x_{i}\right) \beta_{v_{i}, v_{j}} \tilde{g}\left(v_{j}, x_{j}\right)\end{array}\right]$

$+\sum_{j=1}^{n-1}\left(\prod_{k=1, k \neq j}^{n-1} \hat{\pi}_{v_{k}}\left(x_{k}\right)\right) \tilde{g}\left(v_{n}, x_{n}\right) \beta_{v_{n}, v_{j}} \tilde{g}\left(v_{j}, v_{j}\right)$

so the result follows by induction.

It follows immediately from Proposition 2 that the field produced on $\left\{v_{1}, \ldots, v_{N-1}, v_{N}\right\}$ extends the field produced on $\left\{v_{1}, \ldots, v_{N-1}\right\}$. However, it is natural to wonder if the distribution of a subfield is the same as the distribution of the quick simulation field on the corresponding subgraph. Considering the marginal distribution with vertex $v_{1}$ removed, using (3.3) and recalling $\sum_{x_{l}} \tilde{g}\left(v_{l}, x_{l}\right)=0$, we break sum below into:

$i, j \neq l, i=l, j=l$ to find:

$$
\begin{aligned}
& \sum_{x_{l} \in \mathbf{X}_{v_{l}}} \Pi_{v_{1}, \ldots, v_{l-1}, v_{l}, v_{l+1}, \ldots, v_{N}}\left(x_{1}, \ldots, x_{l-1}, x_{l}, x_{l+1}, \ldots, x_{N}\right) \\
& =\prod_{\substack{i=1 \\
i \neq l}}^{N} \pi_{v_{i}}\left(x_{i}\right)+\sum_{\substack{1 \leq j<i \leq N \\
i, j \neq l}}\left[\begin{array}{l}
\left.\left(\sum_{x_{i}} \prod_{k=1, k \neq j}^{i-1} \hat{\pi}_{v_{k}}\left(x_{k}\right) \times \prod_{k=i+1}^{N} \pi_{v_{k}}\left(x_{k}\right)\right)\right] \\
\times \tilde{g}\left(v_{i}, x_{i}\right) \beta_{v_{i}, v_{j}} \tilde{g}\left(v_{j}, x_{j}\right)
\end{array}\right] \\
& +\sum_{1 \leq j<l}\left(\prod_{k=1, k \neq j}^{i-1} \hat{\pi}_{v_{k}}\left(x_{k}\right) \times \prod_{k=i+1}^{N} \pi_{v_{k}}\left(x_{k}\right)\right) \\
& \sum_{x_{l}} \tilde{g}\left(v_{l}, x_{l}\right) \beta_{v_{l}, v_{j}} \tilde{g}\left(v_{j}, x_{j}\right) \\
& +\sum_{1 \leq i<N}\left(\prod_{k=1, k \neq j}^{i-1} \hat{\pi}_{v_{k}}\left(x_{k}\right) \times \prod_{k=i+1}^{N} \pi_{v_{k}}\left(x_{k}\right)\right) \tilde{g}\left(v_{i}, x_{i}\right) \beta_{v_{i}, v_{l}} \sum_{x_{l}} \tilde{g}\left(v_{l}, x_{l}\right) \\
& =\prod_{\substack{i=1 \\
i \neq 1}}^{N} \pi_{v_{i}}\left(x_{i}\right)+\sum_{\substack{1 \leq j<i \leq N \\
i, j \neq l}}\left(\prod_{\substack{k=1 \\
k \neq j, l}}^{i-1} \hat{\pi}_{v_{k}}\left(x_{k}\right) \times \prod_{\substack{k=i+1 \\
k \neq l}}^{N} \pi_{v_{k}}\left(x_{k}\right)\right) \\
& \tilde{g}\left(v_{i}, x_{i}\right) \beta_{v_{i}, v_{j}} \tilde{g}\left(v_{j}, x_{j}\right)
\end{aligned}
$$

This is just the distribution we would have arrived at if we had just simulated $\left\{v_{1}, \ldots, v_{l-1}, v_{l+1}, \ldots, v_{N}\right\}$ in order. Using (3.9) repeatedly, we have proved the following marginality lemma.

\section{Lemma 1}

Suppose the conditions of Proposition 2 hold and $\vec{B} \subset \vec{V}$. Then:

$\Pi_{\vec{B}}\left(x_{\vec{B}}\right)=\sum_{x_{\vec{B}} \in \mathbf{X}_{\bar{B}^{C}}} \Pi_{\vec{V}}(x)$ 


\section{Example 5}

The closed form on $\vec{V}=\{1,2\}$ is:

$$
\Pi_{(1,2)}\left(x_{1}, x_{2}\right)=\pi_{1}\left(x_{1}\right) \pi_{2}\left(x_{2}\right)+\tilde{g}\left(2, x_{2}\right) \beta_{2,1} \tilde{g}\left(1, x_{1}\right)
$$

and the closed form on $\vec{V}=\{1,2,3\}$ is:

$$
\begin{aligned}
\Pi_{(1,2,3)}\left(x_{1}, x_{2}, x_{3}\right)= & \pi_{1}\left(x_{1}\right) \pi_{2}\left(x_{2}\right) \pi_{3}\left(x_{3}\right) \\
& +\pi_{3}\left(x_{3}\right) \tilde{g}\left(2, x_{2}\right) \beta_{2,1} \tilde{g}\left(1, x_{1}\right) \\
& +\hat{\pi}_{2}\left(x_{2}\right) \tilde{g}\left(3, x_{3}\right) \beta_{3,1} \tilde{g}\left(1, x_{1}\right) \\
& +\hat{\pi}_{1}\left(x_{1}\right) \tilde{g}\left(3, x_{3}\right) \beta_{3,2} \tilde{g}\left(2, x_{2}\right)
\end{aligned}
$$

Now, suppose $\beta_{3,1}=\beta_{3,2}=0$ so $X_{1}$ and $X_{2}$ are both uncorrelated from $X_{3}$ by Proposition 2 (a) and:

$$
\begin{aligned}
& \Pi_{(1,2,3)}\left(x_{1}, x_{2}, x_{3}\right) \\
& =\left(\pi_{1}\left(x_{1}\right) \pi_{2}\left(x_{2}\right)+\tilde{g}\left(2, x_{2}\right) \beta_{2,1} \tilde{g}\left(1, x_{1}\right)\right) \pi_{3}\left(x_{3}\right) \\
& =\Pi_{(1,2)}\left(x_{1}, x_{2}\right) \pi_{3}\left(x_{3}\right)
\end{aligned}
$$

by the previous two equations so $X_{3}$ is actually independent of $X_{1}, X_{2}$. The situation is less simple when not considering the last vertex simulated. If $\beta_{3,1}=\beta_{2,1}=$ 0 so $X_{3}$ and $X_{2}$ are both uncorrelated from $X_{1}$ by Proposition 2 (a), then it follows by Lemma 1 that $\prod_{(2,3)}$ $\left(x_{2}, x_{3}\right)=\pi_{2}\left(x_{2}\right) \pi_{3}\left(x_{3}\right)+\tilde{g}\left(3, x_{3}\right) \beta_{3,2} \tilde{g}\left(2, x_{2}\right)$ and:

$$
\begin{aligned}
\Pi_{(1,2,3)}\left(x_{1}, x_{2}, x_{3}\right)= & \pi_{1}\left(x_{1}\right) \Pi_{(2,3)}\left(x_{2}, x_{3}\right) \\
& +\left(\hat{\pi}_{1}\left(x_{1}\right)-\pi_{1}\left(x_{1}\right)\right) \tilde{g}\left(3, x_{3}\right) \beta_{3,2} \tilde{g}\left(2, x_{2}\right)
\end{aligned}
$$

Hence, since each $\hat{\pi}_{v}$ is non-trivial we must either have $\hat{\pi}_{1}=\pi_{1}$ or $\beta_{3,2}=0$ for $X_{1}$ to be independent of $X_{2}, X_{3}$. The case of $X_{2}$ being independent of $X_{1}, X_{3}$ similarly requires $\hat{\pi}_{2}=\pi_{2}$ or $\beta_{3,1}=0$ in addition to $\beta_{3,2}=\beta_{2,1}=0$.

This example illustrates several things about Quick Simulation Fields: order matters in general, there are dependent uncorrelated field and independence generally does not happen when $\hat{\pi}_{v} \neq \pi_{v}$. Indeed, we explain below there is usually dependence even when $\hat{\pi}_{v}=\pi_{v}$.

\section{Example 6}

In the important special case where $\hat{\pi}_{v}=\pi_{v}$ for all $v$ the closed form becomes:

$$
\begin{aligned}
& \Pi_{v_{1}, \ldots, v_{n}}\left(x_{1}, \ldots, x_{n}\right)= \\
& \quad \prod_{l=1}^{n} \pi_{v_{l}}\left(x_{l}\right)\left(1+\sum_{1 \leq j<i \leq n} \frac{\tilde{g}\left(v_{i}, x_{i}\right)}{\pi_{v_{i}}\left(x_{i}\right)} \beta_{v_{i}, v_{j}} \frac{\tilde{g}\left(v_{j}, x_{j}\right)}{\pi_{v_{j}}\left(x_{j}\right)}\right)
\end{aligned}
$$

for each $x_{i} \in \boldsymbol{X}_{v_{i}}$ and $n=1, \ldots, N$.
Now, suppose that $l \in\{1, \ldots N-1\}$ and $\beta_{v_{i}, v_{j}}=0$ when $j \leq l<i$. Then:

$$
\begin{array}{r}
\frac{\Pi_{v_{1}, \ldots, v_{N}}\left(x_{1}, \ldots, x_{N}\right)}{\Pi_{v_{1}, \ldots, v_{l}}\left(x_{1}, \ldots, x_{l}\right) \Pi_{v_{l+1}, \ldots, v_{N}}\left(x_{l+1}, \ldots, x_{N}\right)} \\
=1-\frac{\left(\sum_{1 \leq j<i \leq l} \frac{\tilde{g}\left(v_{i}, x_{i}\right)}{\pi_{v_{i}}\left(x_{i}\right)} \beta_{v_{i}, v_{j}} \frac{\tilde{g}\left(v_{j}, x_{j}\right)}{\pi_{v_{j}}\left(x_{j}\right)}\right)}{\left(1+\sum_{1 \leq j<i \leq l} \frac{\tilde{g}\left(v_{i}, x_{i}\right)}{\pi_{v_{i}}\left(x_{i}\right)} \beta_{v_{i}, v_{j}} \frac{\tilde{g}\left(v_{j}, x_{j}\right)}{\pi_{v_{j}}\left(x_{j}\right)}\right)} \\
\frac{\left(\sum_{l+1 \leq j<i \leq N} \frac{\tilde{g}\left(v_{i}, x_{i}\right)}{\pi_{v_{i}}\left(x_{i}\right)} \beta_{v_{i}, v_{j}} \frac{\tilde{g}\left(v_{j}, x_{j}\right)}{\pi_{v_{j}}\left(x_{j}\right)}\right)}{\left(1+\sum_{l+1 \leq j<i \leq N} \frac{\tilde{g}\left(v_{i}, x_{i}\right)}{\pi_{v_{i}}\left(x_{i}\right)} \beta_{v_{i}, v_{j}} \frac{\tilde{g}\left(v_{j}, x_{j}\right)}{\pi_{v_{j}}\left(x_{j}\right)}\right)}
\end{array}
$$

so one requires:

$$
\begin{gathered}
\sum_{1 \leq j<i \leq l} \frac{\tilde{g}\left(v_{i}, x_{i}\right)}{\pi_{v_{i}}\left(x_{i}\right)} \beta_{v_{i}, v_{j}} \frac{\tilde{g}\left(v_{j}, x_{j}\right)}{\pi_{v_{j}}\left(x_{j}\right)}=0 \text { or } \\
\sum_{l+1 \leq j<i \leq N} \frac{\tilde{g}\left(v_{i}, x_{i}\right)}{\pi_{v_{i}}\left(x_{i}\right)} \beta_{v_{i}, v_{j}} \frac{\tilde{g}\left(v_{j}, x_{j}\right)}{\pi_{v_{j}}\left(x_{j}\right)}=0
\end{gathered}
$$

for independence.

\section{Permutation Property}

Let $V=\left\{v_{i}\right\}_{i=1}^{N}, M_{k}=\{1,2, \ldots, k\}$ for $k \leq N$ and $\boldsymbol{G}_{k}$ be the symmetric group of permutations on $M_{k}$ with composition, denoted $\mathrm{o}$, as group operation, identity permutation $e(i)=i, \forall i \in M_{k}$ and generators $(i i+1)$ in cyclic form for $1 \leq i \leq k-1 .\left\{\left\{v_{a(i)}\right\}_{i=1}^{N}: a \in \boldsymbol{G}_{N}\right\}$ gives the possible simulation orders. We are interested in when the distribution is unchanged.

\section{Definition 1}

Random field $\Pi$ on $V=\left\{v_{i}\right\}_{i=1}^{N}$ the permutation property if:

$$
\Pi_{v_{a(1)}, \ldots, v_{a(N)}}\left(x_{a(1), \ldots,} x_{a(N)}\right)=\Pi_{v_{1}, \ldots, v_{N}}\left(x_{1}, \ldots, x_{N}\right)
$$

for every $\alpha \in \boldsymbol{G}_{N}$.

Marginality then gives:

$$
\Pi_{v_{i_{a(1)}}, \ldots, v_{i_{a(k)}}}\left(x_{i_{a(1)}}, \ldots, x_{i_{a(k)}}\right)=\Pi_{v_{i_{1}}, \ldots, v_{i_{k}}}\left(x_{i_{1}}, \ldots, x_{i_{k}}\right)
$$

for every $1 \leq i_{1}<i_{2}<\ldots<i_{k} \leq N$ and $a \in \boldsymbol{G}_{k}$ when the permutation property holds. 


\section{Theorem 1}

Suppose $N \geq 3, \bar{G}=(V, \bar{E})$ is the completion of connected undirected graph $G=(V, E)$ and $\left\{\pi_{v}\right\}_{v \in V},\left\{\tilde{\pi}_{v}\right\}_{v \in V}$ and $\left\{\hat{\pi}_{v}\right\}_{v \in V}$ are non-degenerate pmfs. Then, in the following (1) and (2) are equivalent and (3) implies (1) and (2):

(1) The permutation property of the $\left\{\pi_{v}, \hat{\pi}_{v}, \tilde{\pi}_{v}, \beta\right\}$-Quick Simulation field $\Pi$ on $\bar{G}$ holds

(2) For each distinct $u, v, w \in V$ :

$$
\begin{aligned}
& \tilde{\sigma}_{w}^{2} \tilde{\pi}_{v}\left(x_{v}\right)\left(x_{v}-\tilde{\mu}_{v}\right) \beta_{v, u}\left(\hat{\pi}_{w}\left(x_{w}\right)-\pi_{w}\left(x_{w}\right)\right) \\
& =\tilde{\sigma}_{v}^{2} \tilde{\pi}_{w}\left(x_{w}\right)\left(x_{w}-\tilde{\mu}_{w}\right) \beta_{w, u}\left(\hat{\pi}_{v}\left(x_{v}\right)-\pi_{v}\left(x_{v}\right)\right)
\end{aligned}
$$

for all $x_{v} \in \boldsymbol{X}_{v}, x_{w} \in \boldsymbol{X}_{w}$.

(3) For each $w \in V$ :

$$
\pi_{\mathrm{w}}\left(x_{w}\right)=\hat{\pi}_{w}\left(x_{w}\right)+c_{w}\left(x_{w}-\tilde{\mu}_{w}\right) \tilde{\pi}_{w}\left(x_{w}\right)
$$

where, for each distinct $u, v, w \in V$ the constants satisfy:

$$
\tilde{\sigma}_{u}^{2} \beta_{w v} c_{u}=\tilde{\sigma}_{v}^{2} \beta_{w u} c_{v}=\tilde{\sigma}_{w}^{2} \beta_{v u} c_{w}
$$

\section{Remark 7}

The following proof reveals the equivalence of (1) and (2) holds even if the original graph $G$ is not connected.

\section{Proof}

$$
\begin{aligned}
& \text { To } \quad \text { ease } \quad \begin{array}{l}
\text { notation, } \\
\boldsymbol{X}_{i}=\boldsymbol{X}_{v_{i}}, \pi_{i}=\pi_{v_{i}}, \hat{\pi}_{i}=\tilde{\pi}_{v_{i}}, \tilde{\pi}_{i}=\tilde{\pi}_{v_{i}}, \beta_{i, j}=\beta_{v_{i}, v_{j}}
\end{array} \text { and: } \\
& y_{i}\left(x_{i}\right)=\hat{\pi}_{i}\left(x_{i}\right)-\pi_{i}\left(x_{i}\right) \forall x_{i} \in \mathbf{X}_{i}, 1 \leq i \leq N
\end{aligned}
$$

For $a \in \boldsymbol{G}_{N}$, one has by commutativity and (3.3) that $X_{a(1)}, \ldots, X_{a(N)}$ has joint pmf:

$$
\begin{gathered}
\prod_{a(1) \ldots, \ldots a(N)}\left(x_{a(1)}, . ., x_{a(N)}\right)=\prod_{i=1}^{N} \pi_{i}\left(x_{i}\right)+ \\
\sum_{1 \leq j<i \leq N}\left(\prod_{k=1, k \neq j}^{i-1} \hat{\pi}_{a(k)}\left(x_{a(k)}\right) \prod_{k=i+1}^{N} \pi_{a(k)}\left(x_{a(k)}\right)\right) \\
\tilde{g}\left(a(i), x_{a(i)}\right) \beta_{a(i), a(j)} \tilde{g}\left(a(j), x_{a(j)}\right)
\end{gathered}
$$

for each $x_{i} \in \boldsymbol{X}_{i}(1 \leq i \leq N)$. By (4.5) the permutation property is equivalent to:

$$
\begin{gathered}
\sum_{1 \leq j<i \leq N}\left(\prod_{k=1, k \neq j}^{i-1} \hat{\pi}_{b(k)}\left(x_{b(k)}\right) \times \prod_{k=i+1}^{N} \pi_{b(k)}\left(x_{b(k)}\right)\right) \times \\
\tilde{g}\left(b(i), x_{b(i)}\right) \beta_{b(i), b(j)} \tilde{g}\left(b(j), x_{b(j)}\right)= \\
\sum_{1 \leq j<i \leq N}\left(\prod_{k=1, k \neq j}^{i-1} \hat{\pi}_{a(k)}\left(x_{a(k)}\right) \times \prod_{k=i+1}^{N} \pi_{a(k)}\left(x_{a(k)}\right)\right) \times \\
\tilde{g}\left(a(i), x_{a(i)}\right) \beta_{a(i), a(j)} \tilde{g}\left(a(j), x_{a(j)}\right)
\end{gathered}
$$

for any two permutations $a, b$.

(1) implies (2): Taking $b=(23)$ o $a$, one finds that the left and right side terms in (4.6) are the same when $j=1, i>$ $3 ; j=2, i=3$ or $j>3$ so, upon cancelling these terms and substituting in for $b$, the remaining $(j=1, i=2 ; j=1 ; i=3 ; j$ $=2, i>3$ and $j=3, i>3$ ) terms in (4.6) become:

$$
\begin{aligned}
& \pi_{a(2)}\left(x_{a(2)}\right) \prod_{k=4}^{N} \pi_{a(k)}\left(x_{a(k)}\right) \tilde{g}\left(a(3), x_{a(3)}\right) \beta_{a(3), a(1)} \tilde{g}\left(a(1), x_{a(1)}\right) \\
& +\hat{\pi}_{a(3)}\left(x_{a(3)}\right) \prod_{k=4}^{N} \pi_{a(k)}\left(x_{a(k)}\right) \tilde{g}\left(a(2), x_{a(2)}\right) \beta_{a(2), a(1)} \tilde{g}\left(a(1), x_{a(1)}\right) \\
& +\hat{\pi}_{a(1)}\left(x_{a(1)}\right) \hat{\pi}_{a(2)}\left(x_{a(2)}\right) \sum_{i=4}^{N} \prod_{k=4}^{i-1} \hat{\pi}_{a(k)}\left(x_{a(k)}\right) \times \\
& \prod_{k=i+1}^{N} \pi_{a(k)}\left(x_{a(k)}\right) \tilde{g}\left(a(i), x_{a(i)}\right) \beta_{a(i), a(3)} \tilde{g}\left(a(3), x_{a(3)}\right) \\
& +\hat{\pi}_{a(1)}\left(x_{a(1)}\right) \hat{\pi}_{a(3)}\left(x_{a(3)}\right) \sum_{i=4}^{N} \prod_{k=4}^{i-1} \hat{\pi}_{a(k)}\left(x_{a(k)}\right) \times \\
& \prod_{k=i+1}^{N} \pi_{a(k)}\left(x_{a(k)}\right) \tilde{g}\left(a(i), x_{a(i)}\right) \beta_{a(i), a(2)} \tilde{g}\left(a(2), x_{a(2)}\right) \\
& =\pi_{a(3)}\left(x_{a(3)}\right) \prod_{k=4}^{N} \pi_{a(k)}\left(x_{a(k)}\right) \tilde{g}\left(a(2), x_{a(2)}\right) \beta_{a(2), a(1)} \tilde{g}\left(a(1), x_{a(1)}\right) \\
& +\hat{\pi}_{a(2)}\left(x_{a(2)}\right) \prod_{k=4}^{N} \pi_{a(k)}\left(x_{a(k)}\right) \tilde{g}\left(a(3), x_{a(3)}\right) \beta_{a(3), a(1)} \tilde{g}\left(a(1), x_{a(1)}\right) \\
& +\hat{\pi}_{a(1)}\left(x_{a(1)}\right) \hat{\pi}_{a(3)}\left(x_{a(3)}\right) \sum_{i=4}^{N} \prod_{k=4}^{i-1} \hat{\pi}_{a(k)}\left(x_{a(k)}\right) \times \\
& \prod_{k=i+1}^{N} \pi_{a(k)}\left(x_{a(k)}\right) \tilde{g}\left(a(i), x_{a(i)}\right) \beta_{a(1), a(2)} \tilde{g}\left(a(2), x_{a(2)}\right) \\
& +\hat{\pi}_{a(1)}\left(x_{a(1)}\right) \hat{\pi}_{a((2)}\left(x_{a(2)}\right) \sum_{i=4}^{N} \prod_{k=4}^{i-1} \hat{\pi}_{a(k)}\left(x_{a(k)}\right) \times \\
& \prod_{k=i+1}^{N} \pi_{a(k)}\left(x_{a(k)}\right) \tilde{g}\left(a(i), x_{a(i)}\right) \beta_{a(i), a(3)} \tilde{g}\left(a(3), x_{a(3)}\right)
\end{aligned}
$$

which simplifies using (4.4) to:

$$
\begin{aligned}
& \tilde{g}\left(a(2), x_{a(2)}\right) \beta_{a(2), a(1)} y_{a(3)}\left(x_{a(3)}\right) \\
& =\tilde{g}\left(a(3), x_{a(3)}\right) \beta_{a(3), a(1)} y_{a(2)}\left(x_{a(2)}\right) .
\end{aligned}
$$

Letting $a$ be such that $a(1)=u, a(2)=v$ and $a(3)=w$, we find (4.1) is necessary.

(2) implies (1): Multiplying (4.1) by $\tilde{g}\left(u, x_{u}\right)$ yields:

$$
\begin{aligned}
& \tilde{g}\left(v, x_{v}\right) \beta_{v, u} \tilde{g}\left(u, x_{u}\right)\left(\hat{\pi}_{w}\left(x_{w}\right)-\pi_{w}\left(x_{w}\right)\right) \\
& =\tilde{g}\left(w, x_{w}\right) \beta_{w, u} \tilde{g}\left(u, x_{u}\right)\left(\hat{\pi}_{v}\left(x_{v}\right)-\pi_{v}\left(x_{v}\right)\right)
\end{aligned}
$$

for all $x_{u} \in \boldsymbol{X}_{u}, x_{v} \in \boldsymbol{X}_{v}, x_{w} \in \boldsymbol{X}_{w}$ and distinct $u, v, w \in M_{N}$. Take $a \in \boldsymbol{G}_{N}$ and let $b=(l l+1)$ o $a$ for $1 \leq l \leq N-1$. Noting that the transpose operations $(l l+1)$ are generators, we just need to show (4.6) for (arbitrary) $a$ and this $b$. However, the left hand terms in (4.6) with $i<l ; j>l+1 ; j \leq$ $l-1, l+2 \leq i$; and $j=l, i=l+1$ directly cancel with the corresponding right hand terms for this $b$. Considering 
the (remaining) terms on the left side of (4.6) with $j \leq l-1$ and $i=l, l+1$ for this $b$ and using (4.9) with $u=a(j), v=$ $a(l), w=a(l+1)$, we get upon manipulation:

$$
\begin{aligned}
& \sum_{j=1}^{l-1} \prod_{\substack{k=1 \\
k \neq j}}^{l-1} \hat{\pi}_{a(k)}\left(x_{a(k)}\right) \prod_{k=l+2}^{N} \pi_{a(k)}\left(x_{a(k)}\right) \tilde{g}\left(a(l+1), x_{a(l+1)}\right) \times \\
& \beta_{a(l+1), a(j)} \tilde{g}\left(a(j), x_{a(j)}\right) \pi_{a(l)}\left(x_{a(l)}\right) \\
& +\sum_{j=1}^{l-1} \prod_{\substack{k=1 \\
k \neq j}}^{l-1} \hat{\pi}_{a(k)}\left(x_{a(k)}\right) \prod_{k=l+2}^{N} \pi_{a(k)}\left(x_{a(k)}\right) \tilde{g}\left(a(l), x_{a(l)}\right) \times \\
& \beta_{a(l), a(j)} \tilde{g}\left(a(j), x_{a(j)}\right) \hat{\pi}_{a(l+1)}\left(x_{a(l+1)}\right) \\
& =\sum_{j=1}^{l-1} \prod_{\substack{k=1 \\
k \neq j}}^{l-1} \hat{\pi}_{a(k)}\left(x_{a(k)}\right) \prod_{k=l+2}^{N} \pi_{a(k)}\left(x_{a(k)}\right) \tilde{g}\left(a(l+1), x_{a(l+1)}\right) \times \\
& \beta_{a(l+1), a(j)} \tilde{g}\left(a(j), x_{a(j)}\right) \hat{\pi}_{a(l)}\left(x_{a(l)}\right) \\
& +\sum_{j=1}^{l-1} \prod_{\substack{k=1 \\
k \neq j}}^{l-1} \hat{\pi}_{a(k)}\left(x_{a(k)}\right) \prod_{k=l+2}^{N} \pi_{a(k)}\left(x_{a(k)}\right) \tilde{g}\left(a(l), x_{a(l)}\right) \times \\
& \beta_{a(l), a(j)} \tilde{g}\left(a(j), x_{a(j)}\right) \pi_{a(l+1)}\left(x_{a(l+1)}\right)
\end{aligned}
$$

so they are equal to the corresponding terms on the right of (4.6). (Notice the switch of $\pi$ and $\hat{\pi}$ in the final factors in (4.10).) Finally, the terms on the left of (4.6) with $j=$ $l, i \geq l+2$ and $j=l+1, i \geq l+2$ for $b=(l l+1) a$ :

$$
\begin{aligned}
& \sum_{i=l+2}^{N}\left(\prod_{\substack{k=1 \\
k \neq l+1}}^{i-1} \hat{\pi}_{a(k)}\left(x_{a(k)}\right) \prod_{k=i+1}^{N} \pi_{a(k)}\left(x_{a(k)}\right)\right) \\
& \tilde{g}\left(a(i), x_{a(i)}\right) \beta_{a(i), a(l+1)} \tilde{g}\left(a(l+1), x_{a(l+1)}\right) \\
& +\sum_{i=l+2}^{N}\left(\prod_{\substack{k=1 \\
k \neq l}}^{i-1} \hat{\pi}_{a(k)}\left(x_{a(k)}\right) \prod_{k=i+1}^{N} \pi_{a(k)}\left(x_{a(k)}\right)\right) \\
& \tilde{g}\left(a(i), x_{a(i)}\right) \beta_{a(i), a(l)} \tilde{g}\left(a(l), x_{a(l)}\right)
\end{aligned}
$$

are just the terms on the right of (4.6) with $j=l+1, i \geq l+2$ and $j=l, i \geq l+2$ i.e. in reverse order. Hence, by breaking the summation up, we have shown (4.6) holds for arbitrary $a$ and $b=(l l+1)$ o $a$, which implies (4.6) holds for arbitrary $a, b$ and sufficiency follows.

\section{(3) Implies (2)}

Letting $u, v, w \in V$ be distinct and using $(4.2,4.3)$, we have that:

$$
\begin{aligned}
& \tilde{\sigma}_{w}^{2} \tilde{\pi}_{v}\left(x_{v}\right)\left(x_{v}-\tilde{\mu}_{v}\right) \beta_{v, u}\left(\hat{\pi}_{w}\left(x_{w}\right)-\pi_{w}\left(x_{w}\right)\right) \\
& =-\tilde{\sigma}_{w}^{2} \tilde{\pi}_{v}\left(x_{v}\right)\left(x_{v}-\tilde{\mu}_{v}\right) \beta_{v, u} c_{w} \tilde{\pi}_{w}\left(x_{w}\right)\left(x_{w}-\tilde{\mu}_{w}\right) \\
& =-\tilde{\sigma}_{v}^{2} \tilde{\pi}_{v}\left(x_{v}\right)\left(x_{v}-\tilde{\mu}_{v}\right) \beta_{w, u} c_{v} \tilde{\pi}_{w}\left(x_{w}\right)\left(x_{w}-\tilde{\mu}_{w}\right) \\
& =\tilde{\sigma}_{v}^{2}\left(\hat{\pi}_{v}\left(x_{v}\right)-\pi_{v}\left(x_{v}\right)\right) \beta_{w, u} \tilde{\pi}_{w}\left(x_{w}\right)\left(x_{w}-\tilde{\mu}_{w}\right)
\end{aligned}
$$

for all $x_{v} \in \mathbf{X}_{v}, x_{w} \in \boldsymbol{X}_{w}$.

In Theorem 1, (1) and (2) almost imply (3), which would establish equivalence. However, the graph must be sufficiently connected as the following example shows.

\section{Example 7}

Suppose $G$ is a connected graph with $N \geq 3$. Suppose further that $G$ is not sufficiently connected. Choose distinct vertices $u, v, w$ so that $u$ and $v$ are neighbors of $w$ and that $u$ and $v$ are not neighbors. The completion of the graph will set $\beta_{v u}=0$ in (4.1), which in turn implies that either $\hat{\pi}_{v}\left(x_{v}\right)-\pi_{v}\left(x_{v}\right)=0 \forall x_{v} \in X_{v}$ or $\beta_{u, w}=0$ on the RHS of (4.1). In the former, $\hat{\pi}_{v}=\pi_{v}$. In the latter, $u$ is not a neighbor of $w$ so only the former is possible. If (3) were true, then $c_{v}=0$ by (4.2) and then $c_{u}=c_{w}=0$ by (4.3) and connectedness.

\section{Theorem 2}

Suppose $\quad N \geq 3, \bar{G}=(V, \bar{E})$ is the completion of sufficiently connected undirected graph $G=(V, E)$; $\left\{\pi_{v}\right\}_{v \in V},\left\{\tilde{\pi}_{v}\right\}_{v \in V}$ and $\left\{\hat{\pi}_{v}\right\}_{v \in V}$ are non-degenerate pmfs with $\left\{\pi_{v} \neq \hat{\pi}_{v}\right\}_{v \in V}$; and (1), (2) and (3) are as in Theorem 1. Then, (1) or (2) imply (3).

Proof. (1) and (2) are equivalent by Theorem 1.

(2) implies (3): Let $u, v, w$ be connected neighbors in $G$. Let $w$ be the neighbor that has two neighbors. This means there are non-zero covariances from $w$ to the other two. (4.1) is (by permuting $u, v, w$ ) equivalent to:

$$
\left[\begin{array}{ccc}
\tilde{g}\left(v, x_{v}\right) \beta_{w v} & -\tilde{g}\left(u, x_{u}\right) \beta_{w u} & \\
& \tilde{g}\left(w, x_{w}\right) \beta_{w u} & -\tilde{g}\left(v, x_{v}\right) \beta_{v u} \\
-\tilde{g}\left(w, x_{w}\right) \beta_{w v} & & \tilde{g}\left(u, x_{u}\right) \beta_{v u}
\end{array}\right]\left[\begin{array}{l}
y_{u}\left(x_{u}\right) \\
y_{v}\left(x_{v}\right) \\
y_{w}\left(x_{w}\right)
\end{array}\right]=0
$$

which implies all solutions have the form:

$$
\begin{aligned}
& y_{u}\left(x_{u}\right)=\frac{\tilde{g}\left(u, x_{u}\right) \beta_{v u}}{\tilde{g}\left(w, x_{w}\right) \beta_{w v}} y_{w}\left(x_{w}\right), \\
& y_{v}\left(x_{v}\right)=\frac{\tilde{g}\left(v, x_{v}\right) \beta_{v u}}{\tilde{g}\left(w, x_{w}\right) \beta_{w u}} y_{w}\left(x_{w}\right)
\end{aligned}
$$

for all $x_{u} \in \boldsymbol{X}_{u}, x_{v} \in \boldsymbol{X}_{\mathrm{v}}, x_{w} \in \boldsymbol{X}_{w}$. This implies that $\frac{y_{w}\left(x_{w}\right)}{\tilde{g}\left(w, x_{w}\right)}$ is constant, which in turn implies:

$$
\begin{aligned}
& \pi_{u}\left(x_{u}\right)=\hat{\pi}_{u}\left(x_{u}\right)+c_{u}\left(x_{u}-\tilde{\mu}_{u}\right) \tilde{\pi}_{u}\left(x_{u}\right) \\
& \pi_{v}\left(x_{v}\right)=\hat{\pi}_{v}\left(x_{v}\right)+c_{v}\left(x_{v}-\tilde{\mu}_{v}\right) \tilde{\pi}_{v}\left(x_{v}\right)
\end{aligned}
$$

where, $c_{u}, c_{v}$ are constants. Since $G$ is sufficiently connected (by non-zero covariances) every vertex can be included in some connected triple as above and we must have that:

$$
\pi_{w}\left(x_{w}\right)=\hat{\pi}_{w}\left(x_{w}\right)+c_{w}\left(x_{w}-\tilde{\mu}_{w}\right) \tilde{\pi}_{w}\left(x_{w}\right)
$$

for all $w \in V$. Now, choosing distinct (not-necessarily connected) $u, v, w \in V$ and using (4.1), we find that these constants must satisfy: $\tilde{\sigma}_{u}^{2} \beta_{w v} c_{u}=\tilde{\sigma}_{v}^{2} \beta_{w u} c_{v}=\tilde{\sigma}_{w}^{2} \beta_{v u} c_{w}$. 


\section{Example 8}

When $N=3$, one $c, c_{u}$ say, can be chosen arbitrarily and the other two can then be solved for by (4.3).

\section{Example 9}

There is always the trivial solution $\hat{\pi}_{u}=\pi_{u}$ (and $\tilde{\pi}_{u}$ arbitrary) for all $u$. This corresponds to taking all the $c_{u}$ to be 0 .

The above theorem gives us the necessary relation:

$$
\pi_{w}\left(x_{w}\right)=\hat{\pi}_{w}\left(x_{w}\right)+c_{w}\left(x_{w}-\tilde{\mu}_{w}\right) \tilde{\pi}_{w}\left(x_{w}\right) \forall w \in V
$$

for the permutation property to hold under sufficient connectivity. Below we will consider completely nontrivial Quick Simulation Fields meaning $\pi_{w} \neq \hat{\pi}_{w}$, i.e. $c_{w} \neq 0$, for all $w \in V$.

We work through an example in a simple four-vertex, black-and-white, complete-graph example, which shows the completely non-trivial solutions. For simplicity (of calculation and simulation) we let all marginal pmfs to be equal, all auxiliary pmfs $\tilde{\pi}_{i}(1)=\tilde{\pi}_{i}(-1)=\frac{1}{2}$ and all covariances to be equal. The fact that the $c_{i}$ 's below can be non-zero means that the solutions can be completely non-trivial. The possible graph distributions are given.

\section{Example 10}

Let $V=\{1,2,3,4\} ; A=\{(1,2),(1,3),(1,4),(2,3),(2$, $4),(3,4)\} ; \boldsymbol{X}_{i}=\{1,-1\}$ for $1 \leq i \leq 4 ; \tilde{\pi}_{i}\left(x_{i}\right)=\frac{1}{2} \forall x_{i} \in \boldsymbol{X}_{i}$; and $\pi_{i}(1)=p$ and $\pi_{i}(-1)=1-p$ where $0<p<1$. It follows that $\tilde{\mu}_{i}=0$ and $\tilde{\sigma}_{i}^{2}=1$. Let $\beta_{12}=\beta_{13}=\beta_{23}=\beta_{14}=\beta_{24}=\beta_{34}=\beta$ so (4.15) holds trivially. It follows by (4.16) that $c_{1}=c_{2}=$ $c_{3}=c_{4}$. Then, $\hat{\pi}_{i}$ must satisfy (4.14) and be a non-trivial pmf for each $i=1,2,3,4$ i.e.:

$$
\hat{\pi}_{i}(1)=\underbrace{\pi_{i}(1)}_{p}-c_{i} \cdot 1 \cdot \underbrace{\tilde{\pi}_{i}(1)}_{\frac{1}{2}}>0
$$

and:

$$
\hat{\pi}_{i}(-1)=1-p+\frac{c_{i}}{2}>0
$$

This translates into the condition $2 p>c_{i}>2 p-2$. The permutable joint pmf of $X_{1}, \ldots, X_{4}$ follows directly from (3.3) as:

$$
\begin{aligned}
& \Pi_{1,2,3,4}\left(x_{1}, x_{2}, x_{3}, x_{4}\right)=\prod_{i=1}^{4} \pi_{i}\left(x_{i}\right) \\
& +\frac{1}{4} \beta\left[\begin{array}{l}
x_{1} x_{2} \pi_{3}\left(x_{3}\right) \pi_{4}\left(x_{4}\right)+x_{1} x_{3} \hat{\pi}_{2}\left(x_{2}\right) \pi_{4}\left(x_{4}\right) \\
+x_{2} x_{3} \hat{\pi}_{1}\left(x_{1}\right) \pi_{4}\left(x_{4}\right)+x_{1} x_{4} \hat{\pi}_{2}\left(x_{2}\right) \hat{\pi}_{3}\left(x_{3}\right) \\
+x_{2} x_{4} \hat{\pi}_{1}\left(x_{1}\right) \hat{\pi}_{3}\left(x_{3}\right)+x_{3} x_{4} \hat{\pi}_{1}\left(x_{1}\right) \hat{\pi}_{2}\left(x_{2}\right)
\end{array}\right]
\end{aligned}
$$

for each $x_{i} \in \boldsymbol{X}(1 \leq i \leq 4)$.

Now, we give our final main result, which establishes necessary and sufficient conditions for completely nontrivial solutions.

\section{Theorem 3}

Suppose $N \geq 4, \bar{G}=(V, \bar{E}) \quad$ is the completion of connected undirected graph $G=(V, E)$ and $\left\{\pi_{v}\right\}_{v \in V},\left\{\tilde{\pi}_{v}\right\}_{v \in V}$ and $\left\{\hat{\pi}_{v}\right\}_{v \in V}$ are non-degenerate pmfs.

Then, the following are equivalent:

a) $G$ is sufficiently connected and disjoint pair rich and there is a completely non-trivial $\left\{\pi_{v}, \hat{\pi}_{v}, \tilde{\pi}_{v}, \beta\right\}$ Quick Simulation field $\Pi$ on $\bar{G}$ satisfying the permutation property

b) The original graph $G$ is complete, (4.14) holds with at least one $c_{w} \neq 0$ and for each distinct $u, v, w, z \in V$ :

$$
\beta_{u, v} \beta_{w, z}=\beta_{u, w} \beta_{v, z}
$$

When (a) and (b) hold, the constants in (4.14) can be taken as:

$$
\begin{aligned}
& c_{v_{1}}=\frac{\tilde{\sigma}_{v_{2}}^{2}}{\tilde{\sigma}_{v_{1}}^{2}} \frac{\beta_{v_{3}, v_{1}}}{\beta_{v_{3}, v_{2}}} c_{v_{2}}, \\
& c_{v_{i+1}}=\frac{\tilde{\sigma}_{v_{i}}^{2}}{\beta_{v_{i+1}, v_{i-1}}} c_{v_{i}} \beta_{v_{i+1}} \beta_{v_{i}, v_{i-1}}
\end{aligned}
$$

where, $c_{v_{2}} \neq 0$ can be taken arbitrarily and $G=\left\{v_{i}\right\}_{i=1}^{N}$.

Proof. (a) implies (b): (4.14) holds by Theorem 2. For distinct $u, v, w, z \in V$, we find by (4.3) that:

$$
\tilde{\sigma}_{u}^{2} \beta_{z u} \beta_{w v} c_{u}=\tilde{\sigma}_{v}^{2} \beta_{z u} \beta_{w u} c_{v}=\tilde{\sigma}_{v}^{2} \beta_{w u} \beta_{z u} c_{v}=\tilde{\sigma}_{u}^{2} \beta_{z v} \beta_{w u} c_{u}
$$

so after cancellation ( $\tilde{\pi}_{u}$ is non-trivial, $c_{u} \neq 0$ ):

$$
\beta_{z u} \beta_{w v}-\beta_{z v} \beta_{w u}=0
$$

Now, suppose $z, u \in V$ that are not neighbors in $G$, we choose distinct $v$ and $w$ to be neighbors of $z$ and $u$ respectively (by disjoint pair rich property). Then, (4.17) implies:

$$
\beta_{z u} \beta_{w v}=\beta_{z v} \beta_{w u} \neq 0 \Rightarrow \beta_{z u} \neq 0
$$

and there is a contradiction. Hence, every $z, u \in V$ are neighbors and $G$ is complete.

(b) implies (a): It follows from completeness and (4.16) that each $c_{i} \neq 0$ :

$$
\tilde{\sigma}_{1}^{2} \beta_{3,2} c_{1}=\tilde{\sigma}_{2}^{2} \beta_{3,1} c_{2}=\tilde{\sigma}_{3}^{2} \beta_{2,1} c_{3}
$$

and: 


$$
\tilde{\sigma}_{i+1}^{2} c_{i+1} \beta_{i, i-1}=\tilde{\sigma}_{i}^{2} c_{i} \beta_{i+1, i-1}=\tilde{\sigma}_{i-1}^{2} c_{i-1} \frac{\beta_{i, i-2} \beta_{i+1, i-1}}{\beta_{i-1, i-2}}
$$

for all $i=3, \ldots, N-1$. However, it follows by (4.15) that:

$$
\beta_{i, i-2} \beta_{i+1, i-1}-\beta_{i-1, i-2} \beta_{i+1, i}=0
$$

so by the previous two equations:

$$
\tilde{\sigma}_{i+1}^{2} c_{i+1} \beta_{i, i-1}=\tilde{\sigma}_{i}^{2} c_{i} \beta_{i+1, i-1}=\tilde{\sigma}_{i-1}^{2} c_{i-1} \beta_{i+1, i}
$$

$\forall i \in\{2, \ldots, N-1\}$.

We have shown (4.3) in the case $u=v_{i-1}, v=v_{i}$ and $w$ $=v_{i+1}$. Now, let $u, v, w \in V$ be arbitrary. Then, they correspond to $v_{i_{3}}, v_{i_{2}}, v_{i_{1}}$ respectively and, without loss of generality, we can assume that $1 \leq i_{1}<i_{2}<i_{3} \leq N$. Using the left hand equality in (4.20) repeatedly, we find:

$$
\begin{aligned}
\tilde{\sigma}_{i_{3}}^{2} c_{i_{3}} \beta_{i_{2}, i_{2}-1} & =\tilde{\sigma}_{i_{3}}^{2} c_{i_{3}} \prod_{j=i_{2}}^{i_{3}-1} \beta_{j, j-1} \frac{1}{\prod_{j=i_{2}+1}^{i_{3}-1} \beta_{j, j-1}} \\
& =\tilde{\sigma}_{i_{2}}^{2} c_{i_{2}} \prod_{j=i_{2}}^{i_{3}-1} \beta_{j+1, j-1} \frac{1}{\prod_{j=i_{2}+1}^{i_{3}-1} \beta_{j, j-1}}
\end{aligned}
$$

However, it follows by repeated use of (4.15) that:

$$
\prod_{j=i_{2}}^{i_{3}-1} \beta_{j+1, j-1}=\beta_{i_{3}, i_{2}-1} \prod_{j=i_{2}+1}^{i_{3}-1} \beta_{j, j-1}
$$

Combining (4.21) and (4.22), one finds:

$$
\tilde{\sigma}_{i_{3}}^{2} c_{i_{3}} \beta_{i_{2}, i_{2}-1}=\tilde{\sigma}_{i_{2}}^{2} c_{i_{2}} \beta_{i_{3}, i_{2}-1}
$$

Moreover, using (4.15) again (when $i_{2}-1 \neq i_{1}$ ), one has that:

$$
\frac{\beta_{i_{2}, i_{2}-1}}{\beta_{i_{3}, i_{2}-1}}=\frac{\beta_{i_{2}, i_{1}}}{\beta_{i_{3}, i_{1}}}
$$

so, substituting (4.24) into (4.23) and relabelling, we have that:

$$
\tilde{\sigma}_{u}^{2} c_{u} \beta_{v, w}=\tilde{\sigma}_{v}^{2} c_{v} \beta_{u, w}
$$

Hence, the first equality in (4.3) holds. The second equality follows in exactly the same manner using the second equality in (4.20) in lieu of the first.

\section{Finding and Conclusion}

We introduced the quick simulation fields, which are correlated Markov fields that can be almost as fast and easy to simulate as independent fields. The given algorithm works on graphs so one can simulate in any dimension and it is an improvement of the prior algorithms of the authors because it facilitates closed form description of the possible field distributions with no added computational costs. The purpose of this work is: (1) To introduce this algorithmic improvement and (2) To discuss important simulation properties that one may wish to hold or wish to avoid. We introduced the following concepts:

(1) Marginality is the property that the induced distribution of a simulated random field on any subfield is the same as the distribution produced by the simulation algorithm directly on the subfield when the simulation order is maintained.

(2) The Permutation Property states that the distribution of a simulated field is the same regardless of the order that the graph vertices are simulated in

(3) A quick simulation field is said to be completely non-trivial if $\pi_{w} \neq \pi_{w}$ for every vertex $w$

(4) A sufficiently connected graph is one where every vertex has a neighbor with two neighbors

(5) A disjoint pair rich graph is one where every pair of non-neighbor vertices have distinct neighbors

Some of our main findings about our quick simulation field are:

(1) There is a closed form for the possible quick simulation field that is given by (3.3).

(2) Marginality always holds by Lemma 1

(3) There are uncorrelated quick simulation fields that are still not independent by Example 5

(4) In the general case, the Permutation Property holds if and only if (4.1) holds

(5) In the sufficiently connected case, the Permutation Property holds if and only if (4.2) and (4.3) both hold

(6) If there are at least four vertices, then the following are equivalent:

(a) The graph is sufficiently connected, disjoint pair rich and there is a completely non-trivial Quick Simulation Field on its completion for any collections $\pi, \tilde{\pi}, \tilde{\pi}, \beta$ (with $\pi_{w} \neq \hat{\pi}_{w}$ for all $w$ ) satisfying the permutation property.

(b) The original graph is already complete, the commutate or type condition (4.15) holds and (4.14) holds with at least one $c_{w} \neq 0$.

\section{Acknowledgement}

This research was sponsored in part by an NSERC Discovery Grant.

\section{Author's Contributions}

All authors equally contributed in this work. 


\section{Ethics}

This article is original and contains unpublished material. The corresponding author confirms that all of the other authors have read and approved the manuscript and there are no ethical issues involved.

\section{References}

Blanchard, P., O. Coulaud and E. Darve, 2016. Fast hierarchical algorithms for generating Gaussian random field. HAL. https://hal.inria.fr/hal-01228519v2

Chellappa, R. and A. Jain, 1993. Markov Random Field: Theory and Application. Ist Edn., Boston: Academic Press, Chellappa, Rama; Jain.

Diaconis, P., 2009. The Markov chain Monte Carlo reolution. Bulletin American Mathematical Society, 46: 179-205.

Kleiber, W., 2016. High resolution simulation of nonstationary Gaussian random field. Comput. Statistics Data Analysis, 101: 277-288.

Kouritzin, M.A., F. Newton and B. Wu, 2013. On random field completely automated public turing test to tell computers and humans apart generation. IEEE Transactions Image Processing, 22: 1656-1666.

Kouritzin, M.A., 2017. Residual and stratified braching partice filters. Comput. Statistics Data Anslysis, 111: 145-165. DOI: 10.1016/j.csda.2017.02.003

Kouritzin, M.A., F. Newton and B. Wu, 2014. A graph theoretic approach to simulation and classification. Computational Statistics Data Analysis, 70: 281-294.
Leblois, E. and J.D. Creutin, 2013. Space-time simulation of intermittent rainfall with prescribed advection field: Adaptation of the turning band method. Water Re-sources Res., 49: 3375-3387.

Li, C., Y. Yuan and R. Wilson, 2008. An unsupervised conditional random field approach for clustering gene expression time series. Bioinformatics, 24: 2467-2473.

Li, H., M. Kallergi, L. Clarke, V. Jain and R. Clark, 1995. Markov random field for tumor detection in digital mammography. IEEE Trans. Medical Imaging, 14: 565-576.

Li, S., 1995. Markov random field modeling in computer vision. Springer-Verlag, Tokyo.

Newton, F. and M.A. Kouritzin, 2011. On grey levels in random CAPTCHA generation. Proceedings of SPIE Visual Information Processing, 8056: 560U-80560U.

Schlather, M., A. Malinowski, P.J. Menck, M. Oesting and K. Strokorb, 2015. Analysis, simulation and prediction of multivariate random fields with package random-fields. J. Statistical Software.

Shinozuka, M. and G. Deodatis, 1996. Simulation of multi-dimensional gaussian stochastic fields by spectral representation. App. Mech. Rev., 49: 29-53.

Vio, R., P. Andreani, L. Tenorio and W. Wamsteker, 2002. Numerical simulation of non-Gaussian random fields with prescribed marginal distributions and crosscorrelation structure II: Multivariate random fields. Publ. Astron. Soc. Pacific, 114: 1281-1289.

Winkler, G., 2003. Image analysis, random fields and Markov chain Monte Carlo methods: A mathematical introduction. 